\title{
A comment concerning cohomology and invariants of Lie algebras with respect to contractions and deformations
}

\author{
R. Campoamor-Stursberg $\dagger$ \\ $\dagger$ Dpto. Geometría y Topología \\ Fac. CC. Matemáticas \\ Universidad Complutense de Madrid \\ Plaza de Ciencias, 3 \\ E-28040 Madrid, Spain \\ E-mail: rutwig@mat.ucm.es
}

\begin{abstract}
Contrary to the expected behavior, we show the existence of noninvertible deformations of Lie algebras which can generate invariants for the coadjoint representation, as well as delete cohomology with values in the trivial or adjoint module. A criterion to decide whether a given deformation is invertible or not is given in dependence of the Poincaré polynomial.
\end{abstract}

PACS numbers: 02.20Sv, 02.20Qs, 98.80Jk 


\section{Introduction}

While contractions were introduced in physics for analyzing the behavior of systems subjected to some limiting process, deformations of Lie algebras and their generalizations entered the theory as an appropriate tool to analyze stability [1. More recent developments, like the attempt to identify the stable form of quantum relativistic kinematical algebras, Quantum Field Theory, expansions of Lie algebras and gauge symmetries or the so called double Special Relativity also head in that direction 2, 3, 4, 5, 6].

Often contractions and deformations are considered as dual operators. However, this idea, although true in some sense, is not entirely satisfactory. It is elementary to prove that any Lie algebra contracts onto the abelian algebra of the same dimension. However, there is no Lie algebra onto which all algebras of the same dimension deform. Thus, although contractions determine a distinguished algebra, the abelian, deformations do not. One could claim that stableł algebras play the analogue role for deformations as the abelian for contractions. However, even this assertion is false since a Lie algebra does not generally deform onto a stable one. In this sense, deformations add new possibilities that cannot appear in limiting processes. Among other properties, contractions of Lie algebras imply various (numerical) relations among invariants of contracting and contracted Lie algebras, such as dimensions of cohomology groups or number of generalized Casimir invariants. It should therefore be expected that deformations imply the reversal of these relations. This must obviously be true for deformations that are the "inverse" of a contraction, but it will be false for a generic deformation.

More specifically, we show that a non-invertible $\oint$ deformation can behave in a rather unexpected way. For example, they can generate Casimir operators and central extensions, which contradict the expected pattern that deformations make a Lie algebra "less abelian". This shows that, although the comparison of invariants of two given Lie algebras provides some information whether a contraction between them is possible, for deformations no assertion can be made by inspection of the corresponding invariants.

\section{Contractions, deformations and cohomology of Lie algebras}

Let $\mathfrak{g}$ be a Lie algebra and $\Phi_{t} \in \operatorname{Aut}(\mathfrak{g})$ a family of automorphisms of $\mathfrak{g}$, where $t \in[1, \infty) . \|$ For any $X, Y \in \mathfrak{g}$ we define

$$
[X, Y]_{\Phi_{t}}:=\Phi_{t}^{-1}\left[\Phi_{t}(X), \Phi_{t}(Y)\right]
$$

$\ddagger$ Also called rigid Lie algebras.

$\S$ Later this concept will be made more precise.

I| Other authors use the parameter range $(0,1]$, which is equivalent to this by simply changing the parameter to $t^{\prime}=1 / t$. 
which obviously are the brackets of the Lie algebra over the transformed basis. Now suppose that the limit

$$
[X, Y]_{\infty}:=\lim _{t \rightarrow \infty} \Phi_{t}^{-1}\left[\Phi_{t}(X), \Phi_{t}(Y)\right]
$$

exists for any $X, Y \in \mathfrak{g}$. Then equation (2) defines a Lie algebra $\mathfrak{g}^{\prime}$ called the contraction of $\mathfrak{g}$ (by $\Phi_{t}$ ), non-trivial if $\mathfrak{g}$ and $\mathfrak{g}^{\prime}$ are non-isomorphic, and trivial otherwise. A contraction for which there exists some basis $\left\{Y_{1}, . ., Y_{n}\right\}$ such that the contraction matrix $A_{\Phi}$ is diagonal, i.e., adopts the form

$$
\left(A_{\Phi}\right)_{i j}=\delta_{i j} t^{n_{j}}, \quad n_{j} \in \mathbb{R}, t>0,
$$

is called a generalized Inönü-Wigner contraction [7]. An important problem in contraction theory, completely solved in [7, is to prove that any contraction is equivalent to a gen. Inönü-Wigner contraction with integer exponents $n_{j}$. This implies that any contraction can be realized by diagonal matrices over some bases.

Deformations of Lie algebras arise from the problem of studying the geometric properties of the variety of Lie algebras when considered as a transformation space. This leads to study the neighborhood of a given point (Lie algebra) in the variety. Deformations are performed using cohomology of Lie algebras [8]. A formal deformation $\mathfrak{g}_{t}$ of a Lie algebra $\mathfrak{g}=(V, \mu)$ is given by the deformed commutator:

$$
[X, Y]_{t}:=[X, Y]+\psi_{m}(X, Y) t^{m}
$$

where $t$ is a parameter and $\psi_{m}: V \times V \rightarrow V$ is a skew-symmetric bilinear map. Imposing the Jacobi identity (up to quadratic order of $t$ ) to the deformed commutator, it follows that the expression satisfied by $\psi_{1}$ characterizes it as a 2-cocycle in the second cohomology space $H^{2}(\mathfrak{g}, \mathfrak{g})$, i.e., it satisfies the constraint

$$
\begin{gathered}
d \psi_{1}\left(X_{i}, X_{j}, X_{k}\right):=\left[X_{i}, \psi_{1}\left(X_{j}, X_{k}\right)\right]+\left[X_{k}, \psi_{1}\left(X_{i}, X_{j}\right)\right]+\left[X_{j}, \psi_{1}\left(X_{k}, X_{i}\right)\right]+ \\
+\psi_{1}\left(X_{i},\left[X_{j}, X_{k}\right]\right)+\psi_{1}\left(X_{k},\left[X_{i}, X_{j}\right]\right)+\psi_{1}\left(X_{j},\left[X_{k}, X_{i}\right]\right)=0 .
\end{gathered}
$$

The preceding computation shows that in order to define a Lie algebra, a deformation has to satisfy an integrability condition.

Definition 1 Let $\varphi \in H^{2}(\mathfrak{g}, \mathfrak{g})$ be a nontrivial cocycle. It is called integrable if it satisfies the condition

$$
\frac{1}{2}[\varphi, \varphi]\left(X_{i}, X_{j}, X_{k}\right):=\sum_{\sigma \in S_{3}} \varphi\left(X_{\sigma(i)}, \varphi\left(X_{\sigma(j)}, X_{\sigma(k)}\right)=0\right.
$$

for all $X_{i}, X_{j}, X_{k}$ in $\mathfrak{g}$.

Therefore, if $\varphi$ is an integrable cocyle, the linear deformation given by

$$
[X, Y]_{t}:=[X, Y]+t \varphi(X, Y)
$$

satisfies the Jacobi identity and defines a Lie algebra. In particular, nullity of $H^{2}(\mathfrak{g}, \mathfrak{g})$ implies that any deformation is isomorphic to $\mathfrak{g}[8]{ }^{+}$

I See apendix A for definitions and properties of cohomology.

+ Such algebras are called cohomologically rigid or stable. 
As known, classical kinematical Lie algebras may be seen as deformations of the static Lie algebra 9]. At the same time, they can be interpreted as contractions of the De Sitter algebras. This suggests that (physically), contractions and deformations are inverse procedures. Although it is not globally true, since there are deformations not related to contractions, any contraction is actually related to a deformation (see e.g. [7]).

Definition 2 A deformation $\mathfrak{g}_{t}(0 \leq t \leq 1)$ is called of plateau type if $\mathfrak{g}_{0} \nsucceq \mathfrak{g}_{1}$ and $\mathfrak{g}_{t} \simeq \mathfrak{g}_{1}$ for all $t \in(0,1]$.

The problem of which deformations are related to a contraction is solved in the following result [7]:

Theorem 1 For any contraction $\mathfrak{g} \rightsquigarrow \mathfrak{g}^{\prime}$ there exists a plateau deformation $\mathfrak{g}^{\prime} \rightarrow \mathfrak{g}$ inverse to the contraction. Conversely, for any deformation of plateau type there exists a contraction inverse to it.

As a consequence, non-invertible deformations are not of plateau type. This result moreover indicates for which class of Lie algebras the invertibility of deformations can fail, namely families of Lie algebras with some parameter that acts as scaling factor on some generators.

\section{Number of invariants and deformations}

Since any Lie algebra contracts onto the abelian Lie algebra $n L_{1}$, in some sense contractions of Lie algebras can be thought of as an "abelianizing" operator. Among other properties, a contraction $\mathfrak{g} \rightsquigarrow \mathfrak{g}^{\prime}$ satisfies the inequalities

$$
\begin{aligned}
& \operatorname{dim} H^{j}(\mathfrak{g}) \leq \operatorname{dim} H^{j}\left(\mathfrak{g}^{\prime}\right), \\
& \operatorname{dim} H^{1}(\mathfrak{g}, \mathfrak{g})<\operatorname{dim} H^{1}\left(\mathfrak{g}^{\prime}, \mathfrak{g}^{\prime}\right), \\
& \operatorname{dim} H^{j}(\mathfrak{g}, \mathfrak{g}) \leq \operatorname{dim} H^{j}\left(\mathfrak{g}^{\prime}, \mathfrak{g}^{\prime}\right), j \neq 1
\end{aligned}
$$

for any $j \geq 0 .{ }^{*}$ This means that any plateau deformation reverses the preceding inequalities. Another important property concerns the number $\mathcal{N}(\mathfrak{g})$ of invariants of the coadjoint representation. Given a basis $\left\{X_{1}, . ., X_{n}\right\}$ of $\mathfrak{g}$ and the structure tensor $\left\{C_{i j}^{k}\right\}$, then $\mathfrak{g}$ can be realized in the space $C^{\infty}\left(\mathfrak{g}^{*}\right)$ by means of the differential operators:

$$
\widehat{X}_{i}=C_{i j}^{k} x_{k} \frac{\partial}{\partial x_{j}},
$$

where $\left[X_{i}, X_{j}\right]=C_{i j}^{k} X_{k} \quad(1 \leq i<j \leq n)$ and $\left\{x_{1}, . ., x_{n}\right\}$ is a dual basis of $\left\{X_{1}, . ., X_{n}\right\}$. The invariants of $\mathfrak{g}$ (in particular, Casimir operators) are the solutions of the system of partial differential equations:

$$
\widehat{X}_{i} F=0, \quad 1 \leq i \leq n .
$$

\footnotetext{
* These identities seem to have been used in the literature without proof. For completeness in the
} exposition, in appendix B we give a proof of these inequalities using exterior forms. 
The number $\mathcal{N}(\mathfrak{g})$ of functionally independent solutions is obtained from the classical criteria for differential equations, and equals:

$$
\mathcal{N}(\mathfrak{g}):=\operatorname{dim} \mathfrak{g}-\operatorname{rank}\left(C_{i j}^{k} x_{k}\right),
$$

where $A(\mathfrak{g}):=\left(C_{i j}^{k} x_{k}\right)$ is the matrix associated to the commutator table of $\mathfrak{g}$ over the given basis. It is known (see e.g. [10]) that for a contraction $\mathfrak{g} \rightsquigarrow \mathfrak{g}^{\prime}$ of Lie algebras, the following inequality must be satisfied

$$
\mathcal{N}(\mathfrak{g}) \leq \mathcal{N}\left(\mathfrak{g}^{\prime}\right)
$$

That is, contractions may generate additional independent invariants for the coadjoint representation. By theorem 1, any deformation of plateau type reverses the preceding inequality. It could therefore be expected that for a deformation $\mathfrak{g}^{\prime} \longmapsto \mathfrak{g}$, even if it not of plateau type, the following inequality is satisfied

$$
\mathcal{N}(\mathfrak{g}) \leq \mathcal{N}\left(\mathfrak{g}^{\prime}\right)
$$

This agrees with the geometric idea that deformations add more components to the structure tensor, and therefore can increase the rank of $A(\mathfrak{g})$. However, we will point out that the latter equation is generally false, which shows that, in general, there is no apparent relation between the number of invariants of a Lie algebra and a deformation which is not of plateau type.

To this extent, let $\mathcal{F}=\left\{\mathfrak{g}_{\alpha}=\mathfrak{s l}(2, \mathbb{R}) \vec{\oplus}_{2 D_{\frac{1}{2}} \oplus D_{0}} A_{5,7}^{1, \alpha, \alpha},-1 \leq \alpha \leq 1\right\}$ be the eight dimensional family of Lie algebras given by the brackets

$$
\begin{array}{llll}
{\left[X_{1}, X_{2}\right]=2 X_{2},} & {\left[X_{1}, X_{3}\right]=-2 X_{3},} & {\left[X_{2}, X_{3}\right]=X_{1},} & {\left[X_{1}, X_{4}\right]=X_{4},} \\
{\left[X_{1}, X_{5}\right]=-X_{5},} & {\left[X_{1}, X_{6}\right]=X_{6},} & {\left[X_{1}, X_{7}\right]=-X_{7},} & {\left[X_{2}, X_{5}\right]=X_{4},} \\
{\left[X_{2}, X_{7}\right]=X_{6},} & {\left[X_{3}, X_{4}\right]=X_{5},} & {\left[X_{3}, X_{6}\right]=X_{7},} & {\left[X_{4}, X_{8}\right]=X_{4},} \\
{\left[X_{5}, X_{8}\right]=X_{5},} & {\left[X_{6}, X_{8}\right]=\alpha X_{6},} & {\left[X_{7}, X_{8}\right]=\alpha X_{7} .} &
\end{array}
$$

For the parameter range $-1 \leq \alpha \leq 1$, these algebras are pairwise non-isomorphic [11]. The parameter $\alpha$ describes the action of a torus on the maximal nilpotent ideal of $\mathfrak{g}_{\alpha}$. Using (8) it is straightforward to verify that

$$
\mathcal{N}\left(\mathfrak{g}_{\alpha}\right)=\left\{\begin{array}{ll}
0, & \alpha \neq-1 \\
2, & \alpha=-1
\end{array} .\right.
$$

Thus $\mathfrak{g}_{-1}$ is a singular element in the family, and deserves a more detailed analysis.

Proposition $1 \mathfrak{g}_{-1}$ is the only member of the family $\mathcal{F}$ that is a contraction of a semisimple Lie algebra.

Proof. Since in dimension 8 the only (real) semisimple Lie algebras are $\mathfrak{s u}(3)$, $\mathfrak{s u}(2,1)$ and $\mathfrak{s l}(3, \mathbb{R})$, if $\mathfrak{g}_{\beta}$ is a contraction of these for some $\beta \in[-1,1]$, then it must satisfy $\mathcal{N}\left(\mathfrak{g}_{\beta}\right) \geq 2$ by (9). Therefore only $\mathfrak{g}_{-1}$ can appear as a contraction. In order to obtain it, we analyze the cohomology group $H^{2}\left(\mathfrak{g}_{-1}, \mathfrak{g}_{-1}\right)$ and the existence of invertible 
(i.e., of plateau type) deformations. A routine buy tedious computation shows that $\operatorname{dim} H^{2}\left(\mathfrak{g}_{-1}, \mathfrak{g}_{-1}\right)=2$, generated by the cocycle classes of

$$
\begin{array}{ll}
\varphi_{1}\left(X_{4}, X_{6}\right)=X_{2}, & \varphi_{1}\left(X_{4}, X_{7}\right)=-\frac{1}{2} X_{1}+\frac{3}{2} X_{8}, \\
\varphi_{1}\left(X_{5}, X_{6}\right)=-\frac{1}{2} X_{1}-\frac{3}{2} X_{8}, & \varphi_{1}\left(X_{5}, X_{7}\right)=-X_{3} . \\
\varphi_{2}\left(X_{6}, X_{8}\right)=X_{6}, \quad \varphi_{2}\left(X_{7}, X_{8}\right)=X_{7} .
\end{array}
$$

Now consider $\mathfrak{g}_{-1}\left(\varepsilon_{1}, \varepsilon_{2}\right)=\mathfrak{g}_{-1}+\varepsilon_{1} \varphi_{1}+\varepsilon_{2} \varphi_{2}$ with bracket operation

$$
\left(\mathfrak{g}_{-1}+\varepsilon_{1} \varphi_{1}+\varepsilon_{2} \varphi_{2}\right)(X, Y):=[X, Y]+\varepsilon_{1} \varphi_{1}(X, Y)+\varepsilon_{2} \varphi_{2}(X, Y) .
$$

It is straightforward to verify that the preceding bracket satisfies the Jacobi condition if and only if $\sharp$

$$
\varepsilon_{1} \varepsilon_{2}=0 .
$$

We therefore obtain two types of linear deformations: $\mathfrak{g}_{-1}\left(\varepsilon_{1}\right):=\mathfrak{g}_{-1}+\varepsilon_{1} \varphi_{1}$ and $\mathfrak{g}_{-1}\left(\varepsilon_{2}\right):=\mathfrak{g}_{-1}+\varepsilon_{2} \varphi_{2}$. Both deformations are integrable and define a Lie algebra. It follows at once that $\left[\mathfrak{g}_{-1}\left(\varepsilon_{1}\right), \mathfrak{g}_{-1}\left(\varepsilon_{1}\right)\right]=\mathfrak{g}_{-1}\left(\varepsilon_{1}\right)$, thus the deformed algebra is perfect. In order to prove that $\mathfrak{g}_{-1}\left(\varepsilon_{1}\right)$ is semisimple for all values of $\varepsilon_{1} \neq 0$, we compute the Killing metric tensor $\kappa$. Over the ordered basis $\left\{X_{1}, . ., X_{8}\right\}$, the matrix of $\kappa$ is given by

$$
A_{\kappa}=\left(\begin{array}{cccccccc}
12 & 0 & 0 & 0 & 0 & 0 & 0 & 0 \\
0 & 0 & 6 & 0 & 0 & 0 & 0 & 0 \\
0 & 6 & 0 & 0 & 0 & 0 & 0 & 0 \\
0 & 0 & 0 & 0 & 0 & 0 & -6 \varepsilon_{1} & 0 \\
0 & 0 & 0 & 0 & 0 & 6 \varepsilon & 0 & 0 \\
0 & 0 & 0 & 0 & 6 \varepsilon_{1} & 0 & 0 & 0 \\
0 & 0 & 0 & -6 \varepsilon_{1} & 0 & 0 & 0 & 0 \\
0 & & 0 & 0 & 0 & 0 & 0 & 4
\end{array}\right) .
$$

For $\varepsilon_{1} \neq 0$ we have $\operatorname{det}\left(A_{\kappa}\right)=-2^{10} 3^{7} \varepsilon_{1}^{4} \neq 0$, thus $\mathfrak{g}_{-1}\left(\varepsilon_{1}\right)$ is semisimple. The eigenvalues of $A_{\kappa}$ are

$$
S p\left(A_{\kappa}\right)=\left\{4,6,-6,12,6 \varepsilon_{1}, 6 \varepsilon_{1},-6 \varepsilon_{1},-6 \varepsilon_{1}\right\},
$$

therefore for any $\varepsilon_{1} \neq 0$ the signature of the matrix is

$$
\sigma=2
$$

proving that $\mathfrak{g}_{-1}\left(\varepsilon_{1}\right)$ is isomorphic to the real normal form $\mathfrak{s l}(3, \mathbb{R})[12$. Moreover, it follows from this proof that $\mathfrak{g}_{-1}\left(\epsilon_{1}\right)$ is a deformation of plateau type, thus we obtain the contraction $\mathfrak{s l}(3, \mathbb{R}) \rightsquigarrow \mathfrak{g}_{1}$ by inversion.

Corollary 1 Any element $\mathfrak{g}_{\alpha}$ of the family $\mathcal{F}$ can be obtained as a linear deformation of $\mathfrak{g}_{-1}$. Additionally, no algebra $\mathfrak{g}_{\alpha}$ contracts nontrivially onto $\mathfrak{g}_{-1}$.

$\sharp$ In this case the obstruction is indeed in the third cohomology group $H^{3}\left(\mathfrak{g}_{-1}, \mathfrak{g}_{-1}\right)$, which has dimension 2. See Table 1. 
Proof. It suffices to consider $0<\varepsilon_{2} \leq 2$. Then $\mathfrak{g}_{-1}\left(\varepsilon_{2}\right)=\mathfrak{g}_{-1}+\varepsilon_{2} \varphi_{2}$ is isomorphic to $\mathfrak{g}_{\varepsilon_{2}-1}$ and we have $-1<\varepsilon_{2}-1 \leq 1$, thus any member of the family can be reached. To show that no member of the family contracts onto $\mathfrak{g}_{-1}$, it suffices to observe that the dimensions of the derivation algebra are:

$$
\operatorname{dim} \operatorname{Der}\left(\mathfrak{g}_{\alpha}\right)=\left\{\begin{array}{cc}
9, & \alpha \neq 1 \\
11, & \alpha=1
\end{array} .\right.
$$

Since for any nontrivial contraction $\mathfrak{g} \rightsquigarrow \mathfrak{g}^{\prime}$ the strict inequality $\operatorname{dim} \operatorname{Der}(\mathfrak{g})<$ $\operatorname{dim} \operatorname{Der}\left(\mathfrak{g}^{\prime}\right)$ must be satisfied, no contraction up to the trivial one is possible. In particular, this shows that no deformation $\mathfrak{g}_{-1}\left(\epsilon_{2}\right)$ is of plateau type.

Proposition 2 For any $-1<\alpha \leq 1$ the Lie algebra $\mathfrak{g}_{-1}$ is a linear deformation of $\mathfrak{g}_{\alpha}$. Moreover the deformation generates two non-constant invariants for the coadjoint representation.

Proof. Computing the adjoint cohomology groups for the family $\mathcal{F}$ (see Table 1 ) it follows that for any value $-1<\alpha \leq 1$ the Lie algebra $\mathfrak{g}_{\alpha}$ admits the nontrivial cocycle $\varphi$ defined by

$$
\varphi\left(X_{6}, X_{8}\right)=X_{6}, \varphi\left(X_{7}, X_{8}\right)=X_{8}
$$

For $\alpha= \pm 1$, this cocycle actually generates the cohomology space $H^{2}\left(\mathfrak{g}_{\alpha}, \mathfrak{g}_{a}\right)$. Now, for any $-1<\alpha \leq 1$ the third cohomology group vanishes, i.e., $H^{3}\left(\mathfrak{g}_{\alpha}, \mathfrak{g}_{\alpha}\right)=0$, which implies that the linear deformation $\mathfrak{g}_{\alpha}(\varepsilon):=\mathfrak{g}_{\alpha}+\varepsilon \varphi$ is integrable and defines a nonisomorphic Lie algebra. Since for any value of $\epsilon$ such that $-1 \leq \alpha+\epsilon \leq 1$ holds the deformed algebra is nonisomorphic, these deformations are never of plateau type, showing that no contractions among the family elements exist. Now, choosing $\epsilon$ such that $\alpha+\varepsilon=-1$, the Lie algebra $\mathfrak{g}_{\alpha}(-1-\alpha)$ has the same commutators as $\mathfrak{g}_{-1}$, thus is isomorphic to it. This proves the first assertion. By (11), $\mathfrak{g}_{\alpha}$ has no invariants for $\alpha \neq-1$, thus the deformation decreases the rank of $A(\mathfrak{g})$ and generates two invariants for the coadjoint representation. $\dagger \dagger$

As a consequence, the deformation decreases the rank of $A\left(\mathfrak{g}_{\alpha}\right)$. This means geometrically that the generic rank of an exterior form in the space spanned by the Maurer-Cartan forms is reduced by the deformation. This fact is of interest for representations, since it indicates the possibility that deformations introduce additional internal labels to describe basis states of a representation [13, 14, 15].

This result implies the general falseness of the intuitive idea that a contraction of Lie algebras "abelianizes" it. The preceding result show that there exist deformations that delete various brackets and make the deformed algebra more "abelian".

$\dagger \dagger$ These two invariants are actually Casimir operators, and can be obtained by contraction of the quadratic and cubic invariants of $\mathfrak{s l}(3, \mathbb{R})$. 


\section{Central extensions and cohomology with trivial coefficients}

It follows from (5) that contractions of Lie algebras can generate cohomology. The physically most useful situation is that a trivial central extension $\mathfrak{g} \otimes \mathbb{R}$ of an algebra $\mathfrak{g}$ leads to a non-trivial central extension $\widehat{\mathfrak{g}}^{\prime}$ of a contraction $\mathfrak{g}^{\prime}$. This happens for example for the Poincaré and Galilei algebras, as well as for other kinematical algebras [5, 9. Obviously deformations of plateau type reverse the inequalities in (5). We illustrate in this section that skipping the assumption of plateau type, no assertions can be made in general on the behavior of the Betti numbers $b_{i}(\mathfrak{g})=\operatorname{dim} H^{i}\left(\mathfrak{g}_{\alpha}\right)$ by deformations.

Proposition 3 Let $-1 \leq \alpha \leq 1$ following relations hold:

(i) For $\alpha \neq-1,0$, the deformations $\mathfrak{g}_{-1} \longrightarrow \mathfrak{g}_{\alpha}$ decrease $b_{2}$ by one unity.

(ii) For $\alpha \neq-1,0$, the deformations $\mathfrak{g}_{\alpha} \longrightarrow \mathfrak{g}_{\beta}(\beta=0,-1)$ increase $b_{2}$ by one unity.

(iii) The deformation $\mathfrak{g}_{-1} \longrightarrow \mathfrak{g}_{0}$ preserves $b_{2}$.

This implies that a general deformation can also create central extensions. By (5), any such deformation is not of plateau type. The proof of this result follows at once by the preceding results and the dimensions of the cohomology groups

$$
\begin{aligned}
& H^{2}\left(\mathfrak{g}_{\alpha}\right)=0, \quad \alpha \neq-1,0, \\
& \operatorname{dim} H^{2}\left(\mathfrak{g}_{\alpha}\right)=1, \alpha=-1,0 .
\end{aligned}
$$

This result has a interesting consequence that allows to determine whether a given deformation can be of plateau type. Recall that for any Lie algebra the Poincaré polynomial is defined as

$$
P_{T}(\mathfrak{g})=1+\sum_{i=1}^{\operatorname{dim} \mathfrak{g}} b_{i}(\mathfrak{g}) T^{i} .
$$

Proposition 4 Let $\mathfrak{g}^{\prime} \longrightarrow \mathfrak{g}$ be a nontrivial deformation. If the polynomial $P_{T}\left(\mathfrak{g}^{\prime}\right)-$ $P_{T}(\mathfrak{g})$ has negative coefficients, then the deformation cannot be of plateau type.

Proof. If the deformation is of plateau type, then it can be reversed to a contraction $\mathfrak{g} \rightsquigarrow \mathfrak{g}^{\prime}$ by theorem 1 . By (15) we have $b_{i}(\mathfrak{g}) \leq b_{i}\left(\mathfrak{g}^{\prime}\right)$ for all $j$. Therefore

$$
P_{T}\left(\mathfrak{g}^{\prime}\right)-P_{T}(\mathfrak{g})=\sum_{i=1}^{\operatorname{dim} \mathfrak{g}}\left(b_{i}\left(\mathfrak{g}^{\prime}\right)-b_{i}(\mathfrak{g})\right) T^{i}
$$

is a polynomial with non-negative coefficients.

We observe that this result can also be applied as a criterion to analyze the existence of contractions. 


\section{Conclusions}

We have seen that in general there is no possible comparison between the cohomology and the number of invariants for a deformation of Lie algebras, up to a special class (plateau type), which correspond exactly to invertible contractions. Specifically we have exhibited a family of Lie algebras which, with two exceptions, does only admit non-invertible deformations. These deformations can create invariants for the coadjoint representation, as well as deleting cohomology, contrary to the expected pattern for deformations. Additionally, these algebras do not deform onto a stable algebra, with the exception of $\mathfrak{g}_{-1}$, thus constitute a singular class of algebras in the variety of Lie algebra laws [8]. They moreover provide a certain geometrical insight for the nonexistence of contractions, since brackets deleted by deformations cannot be recovered by a limiting process.

Geometrically, the notion of non-plateau type deformations adds a new perspective to the non-stability of a system. While plateau deformations give rise to a contraction, thus offering the transition from one system to the other, non-plateau type deformations imply not only instability (like all deformations), but also non-reversibility of processes when subjected to infinitesimal changes. Consider for instance the Bianchi $\mathrm{VII}_{h}$-type algebras (including $\mathrm{VII}_{0}$ type)

$$
\left[X_{1}, X_{2}\right]=h X_{2}+X_{3}, \quad\left[X_{1}, X_{3}\right]=-X_{2}+h X_{3}
$$

Making the appropriate changes of coordinates, the Bianchi metrics can be reduced to the following form

$$
\begin{aligned}
d s^{2}=e^{-2 h x_{3}} & \left(a^{2}(t)\left(\cos x_{3} d x_{1}+\sin x_{3} d x_{2}\right)^{2}+b^{2}(t)\left(\sin x_{3} d x_{1}-\cos x_{3} d x_{2}\right)^{2}\right) \\
& +c^{2}(t) d x_{3}^{2}-d t^{2} .
\end{aligned}
$$

We observe that $\lim _{h \rightarrow 0} d s^{2}$ gives the metric

$d s^{2}=a^{2}(t)\left(\cos x_{3} d x_{1}+\sin x_{3} d x_{2}\right)^{2}+b^{2}(t)\left(\sin x_{3} d x_{1}-\cos x_{3} d x_{2}\right)^{2}+c^{2}(t) d x_{3}^{2}-d t^{2}$,

which actually coincides with the Bianchi metric for type $\mathrm{VII}_{0}$. That is, the metrics of the corresponding models are related by a limiting process, although for no value $h \neq 0$ (15) contracts onto $\mathrm{VII}_{0}$. However, the latter deforms to any Bianchi $\mathrm{VII}_{h}$ type by means of a deformation of non-plateau type. Thus introducing an infinitesimal parameter in $\mathrm{VII}_{0}$ leads to a non-equivalent model with quite different physical properties [16. One of these is that this infinitesimal change in the parameter connects the flat and open Friedmann-Robertson-Walker models [17, and that there is no possibility of recovering the flat model from the open one.

The existence of deformations of non-plateau type could therefore be of some use in physical models depending on some parameters, in order to analyze their sensitiveness to small changes (i.e. perturbations) of the model. These changes could be interpreted as an additional degree of instability of systems, opposed to stable systems associated to rigid (e.g. semisimple) Lie algebras. 
Table 1. Adjoint cohomology of the family $\mathcal{F}$

\begin{tabular}{|c|c|c|c|c|}
\hline$\alpha$ & $\operatorname{dim} H^{3}\left(\mathfrak{g}_{\alpha}, \mathfrak{g}_{\alpha}\right)$ & $\operatorname{dim} H^{2}\left(\mathfrak{g}_{\alpha}, \mathfrak{g}_{\alpha}\right)$ & $\operatorname{dim} \operatorname{Der}\left(\mathfrak{g}_{\alpha}\right)$ & $\mathcal{N}\left(\mathfrak{g}_{\alpha}\right)$ \\
\hline-1 & 2 & 2 & 9 & 2 \\
$\alpha \neq \pm 1$ & 0 & 1 & 9 & 0 \\
1 & 0 & 3 & 11 & 0 \\
\hline
\end{tabular}

\section{Acknowledgement}

The authors wishes to express his gratitude to J. A. de Azcárraga for reference [5] and useful comments.

\section{References}

[1] Vilela Mendes R 1994 J. Phys. A: Math. Gen. 278091

[2] Fadeev L D 1988 Asia-Pacific Physics News 321

[3] Amelino-Camendia G 2002 Int. J. Mod. Phys. D 1135

[4] Chryssomalakos C, Okon E 2004 Int. J. Mod. Phys. D 131817

[5] de Azcárraga J A, Izquierdo J M, Picón M and Varela O 2004 Class.Quant.Grav. 21 S1375

[6] Chaichian M, Presnajder P 2004 Phys.Lett. A 322156

[7] Weimar-Woods E 2000 Rev. Math. Phys. 121505

[8] Nijenhuis A, Richardson R W 1966 Bull. Amer. Math. Soc. 721

[9] Bacry H, Levy-Leblond J M 1968 J. Math. Phys. 91305

[10] Campoamor-Stursberg R 2003 Acta Physica Polonica B 343901

[11] Turkowski P 1988 J. Math. Phys. 292139

[12] Cornwell J F 1984 Group Theory in Physics (New York: Academic)

[13] Racah G 1951 Group Theory and Spectroscopy (Princeton Univ. Press, N. J.)

[14] Campoamor-Stursberg R 2004 Phys. Lett. A 327138

[15] Campoamor-Stursberg R 2006 SIGMA 2 p28

[16] Coley A, Hervik S 2005 Class. Quantum. Grav. 22579

[17] Hervik S, van den Hoogen R J, Lim W C and Coley A A 2006 Class. Quantum. Grav. 23845

[18] de Azcárraga J A, Izquierdo J M 1995 Lie Groups, Lie Algebras, Cohomology and some Applications to Physics (Cambridge: Cambridge Univ. Press) 
A comment concerning cohomology and invariants of Lie algebras

\section{Appendix A. Cohomology of Lie algebras}

Let $\mathfrak{g}$ be a Lie algebra and $V$ a representation of $\mathfrak{g}$. A $p$-dimensional cochain of $\mathfrak{g}$ (with values in $V$ ) is a $p$-linear skew-symmetric mapping

$$
\Phi: \mathfrak{g} \times .^{(p)} \cdot \times \mathfrak{g} \longrightarrow V .
$$

A 0-cochain is by definition a constant function. We denote by $C^{p}(\mathfrak{g}, V)=$ $\operatorname{Hom}\left(\bigwedge^{p} \mathfrak{g}, V\right)$ the space of $p$-cochains. We can provide $C^{p}(\mathfrak{g}, V)$ with the structure of a $\mathfrak{g}$-module structure by putting

$$
(X \Phi)\left(X_{1}, \ldots, X_{p}\right)=X . \Phi\left(X_{1}, \ldots, X_{p}\right)-\sum_{1 \leq i \leq p} \Phi\left(X_{1}, \ldots,\left[X, X_{i}\right], \ldots, X_{p}\right)
$$

for all $X_{1}, \ldots, X_{p} \in \mathfrak{g}$. The coboundary operator $d_{p}: C^{p}(\mathfrak{g}, V) \longrightarrow C^{p+1}(\mathfrak{g}, V)$ is defined by

$$
\begin{aligned}
d_{p} \Phi\left(X_{1}, \ldots, X_{p+1}\right) & =\sum_{1 \leq s \leq p+1}(-1)^{s+1}\left(X_{s} . \Phi\right)\left(X_{1}, \ldots, \widehat{X}_{s}, \ldots, X_{p+1}\right)+ \\
& +\sum_{1 \leq s \leq t \leq p+1}(-1)^{s+t} \Phi\left(\left[X_{s}, X_{t}\right], X_{1}, \ldots, \widehat{X}_{s}, \ldots, \widehat{X}_{t, \ldots}, X_{p+1}\right)
\end{aligned}
$$

By this definition, $d_{p}\left(C^{p}(\mathfrak{g}, \mathfrak{g})\right) \subset C^{p+1}(\mathfrak{g}, \mathfrak{g})$, and it can be verified that $d_{p} \circ d_{p}=0$ for all $p$. The space of $p$-cocycles is defined as $Z^{p}(\mathfrak{g}, V)=\operatorname{ker} d_{p}$, and coboundaries by $B^{p}(\mathfrak{g}, V)=\operatorname{Im} d_{p}$. The $p^{t h}$-cohomology space with values in $V$ is then defined by

$$
H^{p}(\mathfrak{g}, V)=Z^{p}(\mathfrak{g}, V) / B^{p}(\mathfrak{g}, V) .
$$

In particular, for any $p \geq$ we have the following identity:

$$
\operatorname{dim} B^{p+1}(\mathfrak{g}, V)=\operatorname{dim} C^{p}(\mathfrak{g}, V)-\operatorname{dim} Z^{p}(\mathfrak{g}, V)=\operatorname{dim} V\left(\begin{array}{c}
\operatorname{dim} \mathfrak{g} \\
p
\end{array}\right)-\operatorname{dim} Z^{p}(\mathfrak{g}, V) \text {. (A. }
$$

Notation 1 For the trivial module $V=\mathbb{R}$ the notation for the cohomology spaces is simply $H^{p}(\mathfrak{g})$.

We recall the interpretation of some cohomology groups of low order (see e.g. [18] for further details):

(i) $H^{0}(\mathfrak{g}, \mathfrak{g})=Z(\mathfrak{g})$ is the centre of $\mathfrak{g}$.

(ii) $H^{1}(\mathfrak{g}, \mathfrak{g})=\operatorname{Der}(\mathfrak{g})$ is the algebra of derivations.

(iii) $\operatorname{dim} H^{1}(\mathfrak{g})=\operatorname{codim}_{\mathfrak{g}}[\mathfrak{g}, \mathfrak{g}]$.

(iv) $H^{2}(\mathfrak{g})$ is identified with the isomorphism classes of one dimensional central extensions of $\mathfrak{g}$. 


\section{Appendix B. Proof of formula (5)}

Proposition 5 Let $\mathfrak{g} \rightsquigarrow \mathfrak{g}^{\prime}$ be a contraction. Then following inequalities hold:

(i) $\operatorname{dim} H^{k}(\mathfrak{g}, \mathbb{R}) \leq \operatorname{dim} H^{k}\left(\mathfrak{g}^{\prime}, \mathbb{R}\right), j \geq 0$

(ii) $\operatorname{dim} H^{k}(\mathfrak{g}, \mathfrak{g}) \leq \operatorname{dim} H^{k}\left(\mathfrak{g}^{\prime}, \mathfrak{g}\right), j \geq 0$.

Proof. We prove the result for the De Rham cohomology, the argument being analogous for the adjoint cohomology. By Theorem 1, there exists a basis $\left\{X_{1}, . ., X_{n}\right\}$ of $\mathfrak{g}$ over which the contraction is given by the diagonal matrix $T(\varepsilon)_{i j}=\delta_{i}^{j} \varepsilon^{n_{j}}$, with $n_{j} \in \mathbb{Z}$. We compute the cohomology over the transformed basis $\left\{X_{i}^{\prime}=\varepsilon^{n_{i}} X_{i}\right\}$. A basis of $C^{p}(\mathfrak{g}, \mathbb{R})$ is clearly given by the elementary cochains

$$
\varphi_{i_{1} \ldots i_{p}}\left(X_{j_{1}}^{\prime}, \ldots, X_{j_{n}}^{\prime}\right)=\delta_{i_{1}}^{j_{1}} \ldots \delta_{i_{n}}^{j_{n}} .
$$

Taking the dual basis $\left\{\omega_{1}, . ., \omega_{n}\right\}$ of $\left\{X_{1}^{\prime}, . ., X_{n}^{\prime}\right\}$, the cocycle can be identified with the exterior form

$$
\varphi_{i_{1} \ldots i_{p}}=\omega_{i_{1}} \wedge \ldots \wedge \omega_{i_{p}} .
$$

Thus an arbitrary $p$-cochain $\varphi$ is given by a $\mathbb{R}$-linear combination

$$
\varphi=\alpha^{i_{1 . . .} i_{p}} \omega_{i_{1}} \wedge \ldots \wedge \omega_{i_{p}} .
$$

The cocycle condition implies the nullity by the coboundary operator

$$
d \varphi=\alpha^{i_{1} . . i_{p}} d\left(\omega_{i_{1}} \wedge \ldots \wedge \omega_{i_{p}}\right)=0 .
$$

Since we are working on the transformed basis, the Maurer-Cartan equations of $\mathfrak{g}$ have the form:

$$
d \omega_{k}=\varepsilon^{n_{i}+n_{j}-n_{k}} C_{i j}^{k} \omega_{i} \wedge \omega_{j}
$$

Developing (B.3) we obtain

$$
(-1)^{i_{l}-1} \alpha^{i_{1} \ldots i_{p}} \varepsilon^{n_{j_{l}}+n_{k_{k}}-n_{i_{l}}} C_{j_{l} k_{l}}^{i_{l}} \omega_{i_{1}} \wedge . \wedge \wedge \omega_{i_{l-1}} \wedge \omega_{j_{l}} \wedge \omega_{k_{l}} \wedge \omega_{i_{l+1}} \wedge . \wedge \omega_{i_{p}}=0 .
$$

The latter equation is nothing but a linear system in the $\alpha^{i_{1} . ., i_{p}}$ whose coefficients are of the type $\varepsilon^{n_{j_{l}}+n_{k_{k}}-n_{i_{l}}} C_{j_{l} k_{l}}^{i_{l}}$. These coefficients depend both on the structure tensor and the contraction parameter $\epsilon$. The coefficient matrix of (B.5) will be denoted by $A\left(\varepsilon^{n_{i}}\right)$. It follows that $Z^{p}(\mathfrak{g}, \mathbb{R})$ is given by the kernel of this system, thus

$$
\operatorname{dim} Z^{p}(\mathfrak{g}, V)=\left(\begin{array}{c}
\operatorname{dim} \mathfrak{g} \\
p
\end{array}\right)-\operatorname{rank} A\left(\varepsilon^{n_{i}}\right) .
$$

Observe that the coefficient matrix depends on $\varepsilon^{n_{i}}$. Thus taking the limit we have

$$
\operatorname{rank} A\left(\varepsilon^{n_{i}}\right) \geq \operatorname{rank}\left(\lim _{\in \rightarrow 0} A\left(\varepsilon^{n_{i}}\right)\right) .
$$

But $B:=\left(\lim _{\in \rightarrow 0} A\left(\varepsilon^{n_{i}}\right)\right)$ is the coefficient matrix we obtain computing the cohomology after the contraction, thus we get

$$
\left(\begin{array}{c}
\operatorname{dim} \mathfrak{g} \\
p
\end{array}\right)-\operatorname{rank}\left(\lim _{\in \rightarrow 0} A\left(\varepsilon^{n_{i}}\right)\right)=\operatorname{dim} Z^{p}\left(\mathfrak{g}^{\prime}, \mathbb{R}\right)
$$


This proves that $\operatorname{dim} Z^{p}(\mathfrak{g}, \mathbb{R}) \leq \operatorname{dim} Z^{p}\left(\mathfrak{g}^{\prime}, \mathbb{R}\right)$ for any $p$. By (A.5) it follows at once that

$$
\operatorname{dim} B^{p}(\mathfrak{g}, \mathbb{R}) \geq \operatorname{dim} B^{p}\left(\mathfrak{g}^{\prime}, \mathbb{R}\right), p \geq 0
$$

Putting together these inequalities we obtain the chain

$$
\begin{aligned}
& \operatorname{dim} Z^{p}\left(\mathfrak{g}^{\prime}, \mathbb{R}\right)-\operatorname{dim} B^{p}\left(\mathfrak{g}^{\prime}, \mathbb{R}\right) \geq \operatorname{dim} Z^{p}\left(\mathfrak{g}^{\prime}, \mathbb{R}\right)-\operatorname{dim} B^{p}(\mathfrak{g}, \mathbb{R}) \\
& \geq \operatorname{dim} Z^{p}(\mathfrak{g}, \mathbb{R})-\operatorname{dim} B^{p}(\mathfrak{g}, \mathbb{R})
\end{aligned}
$$

that is,

$$
\operatorname{dim} H^{p}\left(\mathfrak{g}^{\prime}, \mathbb{R}\right) \geq \operatorname{dim} H^{p}(\mathfrak{g}, \mathbb{R}) .
$$

As a direct consequence of this formula, no contraction $\mathfrak{g}$ of a semisimple Lie algebra $\mathfrak{s}$ can have vanishing cohomology $H^{3}(\mathfrak{g})$. 